\title{
Early Interventions for Infants with Clinical Signs of Cerebral Palsy
}

\author{
Puneet Wadhwa* \\ Prime Hospital, The Royal College of Pediatrics and Child Health University, UAE \\ *Corresponding author: Puneet Wadhwa, Prime Hospital, The Royal College of Pediatrics and Child Health University, Dubai, UAE
}

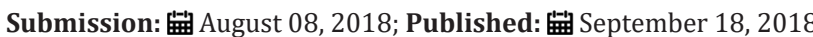

\section{Introduction}

In a bid to construct an all-encompassing definition of the multi-faceted condition that is cerebral palsy (CP), The Study of Cerebral Palsy in Europe (SCPE) and the American Academy for Cerebral Palsy and Developmental Medicine (AACPDM) have attempted a unifying definition, as follows: "Cerebral palsy describes a group of permanent disorders of the development of movement and posture, causing activity limitation, that are attributed to non-progressive disturbances that occurred in the developing foetal or infant brain. The motor disorders are often accompanied by disturbances of sensation, perception, cognition, communication and behavior; by epilepsy and by secondary musculoskeletal problems" [1].

Registers' indicate the average age for the diagnosis of $\mathrm{CP}$ is 19 months. As challenging it is to define $\mathrm{CP}$ we face the same challenge to identify and diagnose it accurately at an early age, although the ability to predict the risk of developing CP at an early age has increased when using a combination of general movement assessments, neurological examinations, and brain imaging techniques $[2,3]$.

There are a wide range of factors, very often in combination, that can be the cause of the early insult to the developing brain that will result in the diagnosis of CP [4-6]. Four distinct groups are at risk of cerebral palsy:

a) Premature infants -whose risk increases as gestational age decreases;

b) Infants with a stroke;

c) Term born infants with neonatal encephalopathy (NE) whose risk of CP increases with increased severity of NE; and

d) "Healthy" term infants born with no identifiable risk factors at birth, but are numerically the largest group of children with cerebral palsy.

To date, there are no biological or pharmacological treatments. However, neurobiological research suggests that the effectiveness of the neural networks and pathways can be strengthened by ear ly interventions. Despite the rather large population of individuals with CP world-wide, there is still a lack of evidence-based early intervention programs that make it possible to utilize the therapeutic window that the rapid brain development and adaptability of the central nervous system during the first year of life represents [2,3].

A plausible reason for the lack of effective early intervention programs for children at risk of developing CP is the common "wait and see" approach due to the uncertainty of early diagnostic criteria [3]. Highly individual developmental trajectories of postural control, motor function, and muscle tone that vary considerably during the first years are frequently seen in children at risk of developing CP. In addition, risk factors and signs of CP might be visible at a very early age but might not necessarily lead to CP. A child with delayed development might recover and atypical signs might disappear, and the opposite can occur in which a child develops CP without obvious early signs. Thus, there is the dilemma in that the potential effect of early intervention might be lost if we wait with intervention until the $\mathrm{CP}$ diagnosis has been confirmed.

Recent neuroplasticity literature suggests that intensive, repetitive, task-specific intervention for CP ought to commence very early while the brain is most plastic, which is almost never the case when "wait and see" monitoring is occurring prior to diagnosis. Plasticity, or neuroplasticity, describes how experiences reorganize neural pathways in the brain. Long lasting functional changes in the brain occur when we learn new things or memorize new information. It is important for those managing the care of infants and young children with motor delay discriminate as early as possible between $\mathrm{CP}$ and other diagnoses.

Early intervention, carried out during the first year in life when the brain is under rapid development and most plastic, is assumed to be more effective than intervention later during development or in adulthood. This assumption is based on the knowledge that neural networks and pathways that remain intact after brain injury can be strengthened through learning-induced plasticity. 
Early intervention (EI) is generally administered as a triad of active motor interventions, parent education and interventions and environmental enrichment.

One such EI program known as The Small Step Program being conducted by a tertiary hospital in Sweden goes one step beyond the traditional management of $\mathrm{CP}$ by identifying infants at risk of CP or other neurodevelopmental disorders and is introduced to such children during their first year of life. The intervention is based upon theories of early learning-induced brain plasticity and comprises important components of evidence-based intervention approaches used with older children with CP.

It is an individualized, goal directed, and intensive intervention that focuses on three key areas: 1) hand use, 2) mobility, and 3) communication. The program is intended to be carried out in the child's home environment and be conducted by their parents who are trained and coached by therapists. The program is designed be used from about 4 months of age and seeks to take advantage of the theoretically plastic period of brain development at this age. The program is designed to include and combine components that are known to be of importance in evidence-based intervention approaches for older children. The challenge in the Small Step Program is to adapt this knowledge and apply it within an intervention for very young children. Another recent EI study is COPing with and CAring for Infants with Special Needs (COPCA). From a family perspective, it is also of interest to highlight that early interventions have been shown to facilitate bonding and to reduce parental stress $[3,7]$.

Based on latest evidence, experts now recommend a shift away from referral for intervention following a formal (most often late) description of $\mathrm{CP}$, to one of referral for intervention which occurs immediately once an infant is considered "at high risk" of CP. With growing evidence regarding available tools and the potential neuroplastic benefits of early intervention, we are heading towards a major change in diagnostic and intervention practice with ultimate aim to ensure that children with disabilities have the same opportunities as all children to reach their development potential and participate meaningfully in their home, school and community environments as children and adults.

\section{References}

1. Fairhurst C (2012) Cerebral palsy: the whys and hows. Arch Dis Child Educ Pract Ed 97(4): 122-131.

2. Skiold B, Eriksson C, Eliasson AC, Aden U, Vollmer B (2013) General movements and magnetic resonance imaging in the prediction of neuromotor outcome in children born extremely preterm. Early Hum Dev 89(7): 467-472.

3. Spittle AJ, Boyd RN, Inder TE, Doyle LW (2009) Predicting motor development in very preterm infants at 12 months' corrected age: the role of qualitative magnetic resonance imaging and general movements assessments. Pediatrics 123(2): 512-517.

4. McIntyre S, Taitz D, Keogh J, Goldsmith S, Badawi N, et al. (2013) A systematic review of risk factors for cerebral palsy in children born at term in developed countries. Dev Med Child Neurol 55(6): 499-508.

5. Keogh JM, Badawi N (2006) The origins of cerebral palsy. Curr Opin Neurol 19(2): 129-134.

6. Bax M, Tydeman C, Flodmark O (2006) Clinical and MRI correlates of cerebral palsy: the European Cerebral Palsy Study. JAMA 296(13): 16021608.

7. Whittingham K, Wee D, Boyd R (2011) Systematic review of the efficacy of parenting interventions for children with cerebral palsy. Child Care Health Dev 37(4): 475-483.
cC (9) Creative Commons Attribution 4.0 International License

For possible submissions Click Here
Submit Article

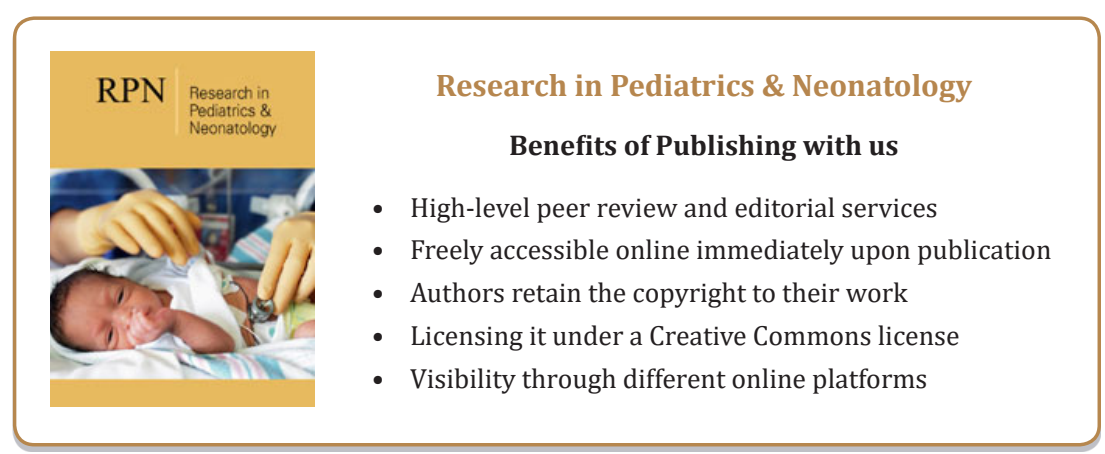

